\title{
Correction
}

\section{Correction to: The Full Ward-Takahashi Identity for Colored Tensor Models}

\section{Carlos I. Pérez-Sánchez $\mathbb{D}$}

Mathematisches Institut, Westfälische Wilhelms-Universität, Einsteinstraße 62, 48149 Münster, Germany. E-mail: perezsan@uni-muenster.de

Received: 8 October 2018 / Accepted: 8 October 2019

Published online: 1 February 2020 - (C) Springer-Verlag GmbH Germany, part of Springer Nature 2020

\section{Correction to: Commun. Math. Phys. 358, 589-632 (2018) https://doi.org/10.1007/s00220-018-3103-2}

The author wishes to rectify some oversights and, mainly, to amend an erroneous term of the analytic 2-point Schwinger-Dyson Equation (SDE) for the rank-3, complex tensor field $\varphi_{3}^{4}$-theory, derived in [Pér18a]. We tag the corrections using primed numeration.

Lemma 4 is stronger than it was stated, and this stronger version is needed. The correct (and rephrased) version is:

Lemma 4'. Let $\mathcal{R}, \mathcal{P} \in \mathrm{Grph}_{\mathrm{c}, 3}$ be connected graphs with $2 p=|V(\mathcal{P})|$ and $2 r=$ $|V(\mathcal{R})|$ vertices, respectively. Let $\mathbf{z}^{1}, \ldots, \mathbf{z}^{r} \in \mathbb{Z}^{3}$ be arbitrary momenta, and let $\mathbf{x}^{1}, \ldots$, $\mathbf{x}^{p} \in \mathbb{Z}^{3}$ be colorwise, pairwise different momenta. That is, for each $\alpha, v=1, \ldots, p$, and for each color $c=1,2,3$, one imposes $x_{c}^{\alpha} \neq x_{c}^{v}$ if $\alpha \neq v$. Then, the derivative of $\mathcal{R}$ with respect to $\mathcal{P}$ satisfies

$$
\frac{\partial \mathcal{R}\left(\mathbf{z}^{1}, \ldots, \mathbf{z}^{r}\right)}{\partial \mathcal{P}\left(\mathbf{x}^{1}, \ldots, \mathbf{x}^{p}\right)}=\left\{\begin{array}{cl}
\delta_{p}^{r} \times\left(\sum_{\sigma \in \operatorname{Aut}_{\mathrm{c}}(\mathcal{R})} \delta_{\mathbf{x}^{1}, \mathbf{x}^{2}, \ldots, \mathbf{x}^{r}}^{\mathbf{z}^{\sigma(1)}, \ldots, \mathbf{z}^{\sigma(r)}}\right) & \text { if } \mathcal{R} \cong \mathcal{P}, \\
0 & \text { if } \mathcal{R} ¥ \mathcal{P} .
\end{array}\right.
$$

That is, the imposed restriction ${ }^{1} z_{c}^{\alpha} \neq z_{c}^{v}$, for $\alpha \neq v$ and $c=1,2,3$, was superfluous (and is not used in the proof). Lemma $4^{\prime}$ ensures that one can derive the free energy $\log Z$ with respect to graphs. The reason is that in the boundary-graph expansion [Eq. (35)] appear terms $\mathbb{J}(\mathcal{R})\left(\mathbf{z}^{1}, \ldots, \mathbf{z}^{r}\right)$ of unrestricted momenta, which correspond to $\mathbf{z}^{1}, \ldots, \mathbf{z}^{r} \in \mathbb{Z}^{3}$ in Eq. $\left(27^{\prime}\right)$ here.

For example, recalling that momenta label white vertices, the equation [Pér18a, before Eq. (33)]

The original article can be found online at https://doi.org/10.1007/s00220-018-3103-2.

\footnotetext{
1 Appearing in [Pér18a, Lemma 4] as "We impose analogous conditions on $\mathbf{c}^{1}, \ldots, \mathbf{c}^{r} \in \mathbb{Z}^{3}$ ".
} 


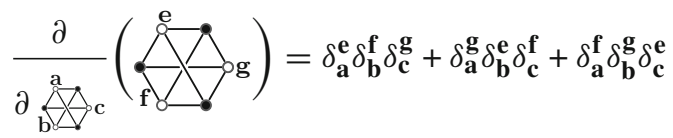

holds everywhere where $\left\{a_{d} \neq b_{d} \neq c_{d} \neq a_{d}\right\}_{d=1,2,3}$, that is, with no restriction on $\mathbf{e}, \mathbf{f}, \mathbf{g} \in \mathbb{Z}^{3}$.

The main correction concerns the 2-point SDE [Pér18a, Eq. (51)], which should be instead:

Claim 1. The exact, analytic Schwinger-Dyson equation for the 2-point function $G^{(2)}$ of the $\varphi_{3}^{4}$-theory, which is defined by the action

$$
S[\varphi, \bar{\varphi}]=\sum_{\mathbf{x}} \bar{\varphi}_{\mathbf{x}} \cdot\left(x_{1}^{2}+x_{2}^{2}+x_{3}^{2}+m^{2}\right) \cdot \varphi_{\mathbf{x}}+\lambda\left(1 \mathcal{D}_{1}+2 \mathscr{D}_{2}+3 \mathscr{D}_{3}\right),
$$

is given by

$$
\begin{aligned}
G^{(2)}(\mathbf{x})= & \frac{1}{m^{2}+|\mathbf{x}|^{2}}+\frac{(-2 \lambda)}{m^{2}+|\mathbf{x}|^{2}} \sum_{c=1}^{3}\left\{\sum_{q_{a}, q_{b}} G^{(2)}\left(q_{a}, q_{b}, x_{c}\right) \times G^{(2)}(\mathbf{x})\right. \\
& +\sum_{d=a, b} \sum_{q_{c}} G_{V_{d}}^{(4)}\left(x_{a}, x_{b}, q_{c}, \mathbf{x}\right)+\sum_{q_{a}, q_{b}} G_{\mathrm{m} \mid \mathrm{m}}^{(4)}\left(q_{a}, q_{b}, x_{c}, \mathbf{x}\right) \\
& \left.-\sum_{q_{c}} \frac{1}{q_{c}^{2}-x_{c}^{2}}\left[G^{(2)}\left(x_{a}, x_{b}, q_{c}\right)-G^{(2)}(\mathbf{x})\right]+G_{V_{c}}^{(4)}(\mathbf{x}, \mathbf{x})\right\},
\end{aligned}
$$

for each $\mathbf{x}=\left(x_{1}, x_{2}, x_{3}\right) \in \mathbb{Z}^{3}$. In $\left(51^{\prime}\right)$, the set equality $\{a, b, c\}=\{1,2,3\}$ is assumed

Here, we conveniently abbreviated:

$$
\begin{aligned}
G^{(2)} & =G_{\ominus}^{(2)}=\frac{\partial \log Z}{\partial \theta}, \quad G_{V_{c}}^{(4)}=G_{c \mid g c}^{(4)}=\frac{\partial \log Z}{\partial c \mid g c}, \\
G_{\mathrm{m} \mid \mathrm{m}}^{(4)} & =G_{\ominus \mid \ominus}^{(4)}=\frac{\partial \log Z}{\partial(\ominus \mid \ominus)} .
\end{aligned}
$$

Unfolding the colors, the correct 2-point SDE $\left(51^{\prime}\right)$ reads $^{2}$ :

$$
\begin{aligned}
& G_{\ominus}^{(2)}(\mathbf{x})=\frac{1}{m^{2}+|\mathbf{x}|^{2}} \\
& +\frac{(-2 \lambda)}{m^{2}+|\mathbf{x}|^{2}}\left\{G_{\ominus}^{(2)}(\mathbf{x}) \cdot\left[\sum_{q, r \in \mathbb{Z}} G_{\ominus}^{(2)}\left(x_{1}, q, r\right)+G_{\ominus}^{(2)}\left(q, x_{2}, r\right)+G_{\ominus}^{(2)}\left(q, r, x_{3}\right)\right]\right. \\
& +\sum_{q \in \mathbb{Z}}\left(G_{1 g_{1}}^{(4)}\left(x_{1}, q, x_{3} ; \mathbf{x}\right)+G_{1 g_{1}}^{(4)}\left(x_{1}, x_{2}, q ; \mathbf{x}\right)+G_{2 g_{2}}^{(4)}\left(q, x_{2}, x_{3} ; \mathbf{x}\right)\right.
\end{aligned}
$$

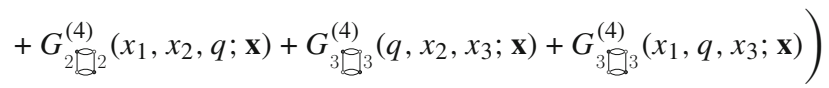

\footnotetext{
2 Here, given in the same notation of [Pér18a] to ease comparison.
} 


$$
\begin{aligned}
& +\sum_{q, r \in \mathbb{Z}}\left[G_{\ominus}^{(4)}\left(x_{1}, q, r ; \mathbf{x}\right)+G_{\ominus}^{(4)}\left(q, x_{2}, r ; \mathbf{x}\right)+G_{\ominus}^{(4)}\left(q, r, x_{3} ; \mathbf{x}\right)\right] \\
& -\sum_{q_{1} \in \mathbb{Z}}\left[\frac{1}{q_{1}^{2}-x_{1}^{2}} \cdot\left(G_{\ominus}^{(2)}\left(q_{1}, x_{2}, x_{3}\right)-G_{\ominus}^{(2)}(\mathbf{x})\right)\right] \\
& -\sum_{q_{2} \in \mathbb{Z}}\left[\frac{1}{q_{2}^{2}-x_{2}^{2}} \cdot\left(G_{\ominus}^{(2)}\left(x_{1}, q_{2}, x_{3}\right)-G_{\ominus}^{(2)}(\mathbf{x})\right)\right] \\
& \left.-\sum_{q_{3} \in \mathbb{Z}}\left[\frac{1}{q_{3}^{2}-x_{3}^{2}} \cdot\left(G_{\ominus}^{(2)}\left(x_{1}, x_{2}, q_{3}\right)-G_{\ominus}^{(2)}(\mathbf{x})\right)\right]+\sum_{c=1,2,3} G_{c \mid g c}^{(4)}(\mathbf{x}, \mathbf{x})\right\} .
\end{aligned}
$$

for all $\mathbf{x}=\left(x_{1}, x_{2}, x_{3}\right) \in \mathbb{Z}^{3}$.

The mistake, which is fixed by replacing the second row of Eq. (51) by the second row of Eq. $\left(51^{\prime}\right)$ (or by the third and fourth lines of Eq. (51")), is a consequence of a computational oversight in page 626 while directly calculating $\delta_{a_{1} b_{1}} \times\left(\delta^{2} Y^{(1)}[J, \bar{J}] / \delta J_{\mathbf{a}} \delta \bar{J}_{b_{1} a_{2} a_{3}}\right)$. In fact, if one uses Eq. (48) $\left.\right|_{a=1}$ for the $Y$-term instead, one gets the correct result for this double derivative, after using the symmetry of each 4-point function:

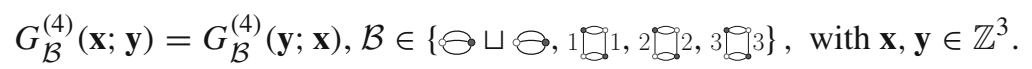

To wit:

$$
\begin{aligned}
& \left.\frac{\delta^{2} Y_{x_{1}}^{(1)}[J, \bar{J}]}{\delta J_{x_{1} x_{2} x_{3}} \delta \bar{J}_{x_{1} x_{2} x_{3}}}\right|_{J=\bar{J}=0}=\frac{\partial Y_{x_{1}}^{(1)}[J, \bar{J}]}{\partial \theta(\mathbf{x})} \\
& =\left(\Delta_{x_{1}, 1} G_{\theta_{0} \ominus^{(4)}}+\Delta_{x_{1}, 1} G_{1 \mathrm{~g}_{1}}^{(4)}+\Delta_{x_{1}, 1} G_{2 g_{2}}^{(4)}+\Delta_{x_{1}, 1} G_{3 \mathrm{~g}_{3}}^{(4)}\right)(\mathbf{x}) \\
& =\sum_{q_{2}, q_{3}} G_{\theta_{0} \ominus^{(4)}}\left(x_{1}, q_{2}, q_{3} ; \mathbf{x}\right)+G_{1 g_{1}}^{(4)}(\mathbf{x}, \mathbf{x}) \\
& +\sum_{q_{3}} G_{2 g_{2}}^{(4)}\left(x_{1}, x_{2}, q_{3} ; \mathbf{x}\right)+\sum_{q_{2}} G_{3 g_{3}}^{(4)}\left(x_{1}, q_{2}, x_{3} ; \mathbf{x}\right) \text {, }
\end{aligned}
$$

with analogous results for $\partial Y_{x_{2}}^{(2)} / \partial \theta$ and $\partial Y_{x_{3}}^{(3)} / \partial \theta$. The second row of Eq. (51) derives from terms in the sum $\sum_{c=1,2,3} \partial Y_{x_{c}}^{(c)} / \partial \partial_{(\mathbf{x}}$, and the correct result is concretely obtained by the last line of Eq. (b) plus cyclic permutations, namely $\sum_{c=1,2,3} \sum_{d \neq c} \sum_{q_{c}} G_{V_{d}}^{(4)}$ $\left(x_{a}, x_{b}, q_{c}, \mathbf{x}\right)$, after some rearrange. Claim 1 follows.

The extraction of the coefficients of the $Y$-term via graph derivatives is explored in depth in [PPW17], whose advantage over direct computations, we hope, is now clear.

To conclude this corrigendum, in page $625 \mathrm{I}$ stated that if for certain color $a=$ $1, \ldots, D$ the subgraph

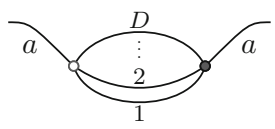


is contained in each one of the interaction vertices of a complex tensor model, then one could obtain the Schwinger-Dyson equations departing from the present WardTakahashi identity. As it reads, this implies that our techniques work for the interaction vertex

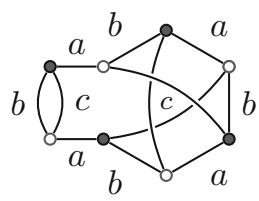

Nevertheless, as it was pointed out to me (see acknowledgements), the previous condition turns out to be necessary but insufficient. The interaction vertices should be furthermore restricted to graphs in which each (graph-)vertex sits in such a subgraph. For instance, on top of the 'pillow-like' (quartic) vertices treated here, one can derive the tower of analytic Schwinger-Dyson equations for interactions spanned by

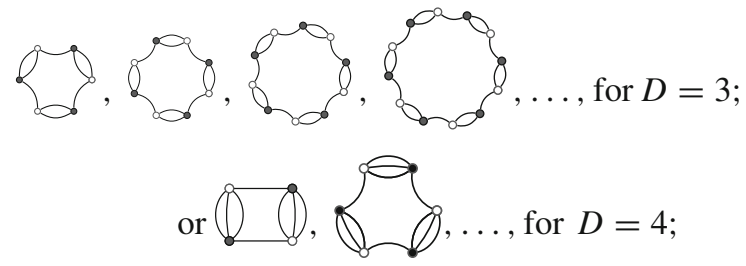

and higher-rank analogues. In fact, the complete tower of SDE for connected correlation functions with connected boundary graphs has been obtained for the arbitrary-rank 'pillow-like' vertices in [PPW17] (for disconnected boundary graphs cf. [Pér18b]). Their large- $N$ limit was later reported in [PPTW18] for rank-3 theories. Moreover, using the Ward-Takahashi identity and extending the approach [PW19] to solve a quartic model in noncommutative field theory in terms of the Lambert $\mathrm{W}$ function, the solution of a single pillow-vertex model [Pas19] was found.

On that basis, the SDE for tensor models with the $\varphi^{n}$-interaction vertices $(n \geq 6)$ listed in (e) could be also derived. How to get the analytic SDE-tower for interactions like (d) is still unknown.

Acknowledgements. Johannes Thürigen and Romain Pascalie are acknowledged for pointing me out the imprecise formulation of the statement in page 625 about the subgraph condition (c). The author was supported first by the Deutsche Forschungsgemeinschaft (SFB 878) and subsequently by the TEAM programme of the Foundation for Polish Science co-financed by the European Union under the European Regional Development Fund (POIR.04.04.00-00-5C55/17-00).

Publisher's Note Springer Nature remains neutral with regard to jurisdictional claims in published maps and institutional affiliations.

\section{References}

[Pér18a] Pérez-Sánchez, C.I.: The full Ward-Takahashi Identity for colored tensor models. Commun. Math. Phys. 358(2), 589-632 (2018)

[PPW17] Pascalie, R., Pérez-Sánchez, C.I., Wulkenhaar, R.: Correlation functions of U( $N)$-tensor models and their Schwinger-Dyson equations (to appear). In: Annales de l'Institut Henri Poincare (D) Combinatorics, Physics and their Interactions. arXiv:1706.07358 (2017) 
[Pér18b] Pérez-Sánchez, C.I.: Graph calculus and the disconnected-boundary Schwinger-Dyson equations of quartic tensor field theories. arXiv:1812.00623 (2018)

[PPTW18] Pascalie, R., Pérez-Sánchez, C.I., Tanasă, A., Wulkenhaar, R.: On the large $N$ limit of SchwingerDyson equations of a rank-3 tensor field theory. J. Math. Phys. 60, 073502 (2019)

[PW19] Panzer, E., Wulkenhaar, R.: Lambert-W solves the noncommutative $\Phi^{4}$-model. Commun. Math. Phys. (2019)

[Pas19] Pascalie, R.: A solvable tensor field theory. arXiv:1903.02907 (2019)

Communicated by M. Salmhofer 